\title{
SPRECHSTUNDE NATURHEILKUNDE
}

\section{Serie}

\section{Angst und Spannungszustände}

\author{
Angst und Spannungszustände werden vielfach mit Anxiolytika und \\ Sedativa behandelt. Diese Medikamente haben jedoch Nebenwirkungen \\ wie Mundtrockenheit oder Sedierung und ein Abhängigkeits- und \\ Gewöhnungspotenzial, weshalb sie von den Patienten oft abgelehnt \\ werden. Die Naturheilkunde kennt eine Reihe von Alternativen, die bei \\ Bedarf kombiniert werden können. Bei schweren Angstzuständen sollte \\ allerdings eine Therapie durch den Psychiater erfolgen.
}

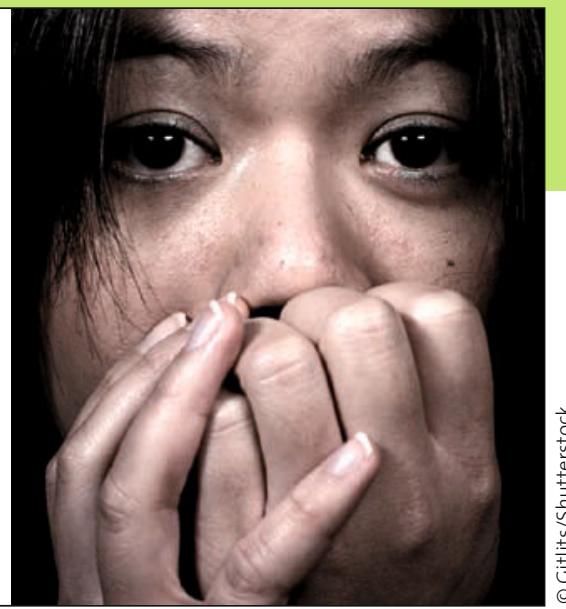

\begin{abstract}
Atemtherapie nach Middendorf
Diese Therapie ist ein übendes, aktives, nicht suggestives, eutonisierendes, selbsterfahrungszentriertes Verfahren, das sich u.a. zur Behandlung von psychovegetativen Spannungszuständen und Phobien eignet. Wichtig für den Therapieerfolg ist die Fähigkeit des $\mathrm{Pa}$ tienten zur Selbstreflektion und die Bereitschaft, sich mit seiner Krankheit auseinanderzusetzen.
\end{abstract}

\section{Entspannungsverfahren}

Das Autogene Training eignet sich zur symptomatischen Mitbehandlung von nervösen Erregungs- und Angstzuständen. Auch die Progressive Relaxation nach Jacobson kann adjuvant eingesetzt werden.

\section{Physikalische Therapie}

Tägliche Ganzwaschungen sowie Armund Beingüsse wirken vegetativ stabilisierend. Die beste Form der Bewegungstherapie sind Spaziergänge in Begleitung. Klimawechsel wirken unterstützend.

\section{Von Baldrian bis Lavendel}

Bei den Extrakten aus Baldrianwurzel, Hopfenzapfen, Melissenblättern, Passionsblumenkraut, bisher als milde Hypnotika eingestuft, wurden neuerdings anxiolytische und spannungsmindernde Wirkungen gefunden. Jedes dieser Phytotherapeutika hat sein eigenes Wirk- profil, Kombinationsarzneimittel sind der Monotherapie offenbar überlegen.

Zudem ist ein Präparat mit patentiertem Lavendelöl (80 mg) erhältlich. Es hat sich in zwei randomisierten Doppelblindstudien bei Patienten mit subsyndromaler Angst bzw. Unruhe und Schlafstörungen hinsichtlich Anxiolyse bereits nach zwei Wochen dem Placebo statistisch signifikant überlegen gezeigt (HAMA-Score, $80 \mathrm{mg} / \mathrm{d}$ ). In einer referenzkontrollierten Studie mit diesem Präparat $(80 \mathrm{mg} / \mathrm{d})$ entsprachen die anxiolytische Wirkung und die Verbesserung der Lebensqualität nach sechs Wochen der von $0,5 \mathrm{mg} / \mathrm{d}$ Lorazepam.

\section{Auch Folgezustände der Angst gebessert}

Phytotherapie eignet sich zur Therapie von leichten bis mittelschweren Angstzuständen v. a., wenn eine mittel- bis langfristige Behandlung erforderlich ist. Nicht nur die Angst selbst, sondern auch Folgezustände der Angst können gebessert werden, z. B. Niedergeschlagenheit und Resignation, Merk- und Konzentrationsstörungen, Reizbarkeit, aggressive Tendenzen sowie vegetative Symptome wie funktionelle Beschwerden. Bis zum vollständigen Wirkungseintritt vergeht mindestens eine Woche.

Zur Behandlung von Angst sollten Kombinationspräparate von Baldrianwurzel mit Hopfenzapfen, Melissenblättern oder Passionsblumenkraut ver- wendet werden. Die Tagesdosis beträgt in Kombinationspräparaten für Baldrianwurzel bis $1500 \mathrm{mg}$, für Hopfen bis 130 mg, für Passionsblume bis 1000 mg und für Melissenblätter bis $270 \mathrm{mg}$ Trockenextrakt. Passionsblumenkraut kann, da es deutlich anxiolytisch wirkt, bei dieser Indikation auch allein verwendet werden $(1200 \mathrm{mg} / \mathrm{d}$ Trockenextrakt). Bei Lavendelöl mit aufkonzentrierten Inhaltsstoffen beträgt die empfohlene Dosis $80 \mathrm{mg} / \mathrm{d}$, die Wirkung tritt nach 14 Tagen ein. Bei Angstzuständen während des Klimakteriums eignet sich Cimicifugawurzelstock, evtl. in Kombination mit Johanniskrautextrakt.

\section{Kaum Nebenwirkungen}

Spezifische Nebenwirkungen, Kontraindikationen und Interaktionen sind für Baldrianwurzel, Hopfenzapfen, Melissenblätter und Passionsblumen nicht beschrieben. Kombinationen aus Baldrianwurzel- und Passionsblumenextrakt sollten wegen einer möglichen Verstärkung der Wirkung von GABAergen Arzneimitteln nicht mit diesen kombiniert werden. Bei Lavendelöl werden bei bis zu 7,5\% der Patienten Aufstoßen sowie in 5\% der Fälle Dyspepsie beobachtet.

Die genannten Phytotherapeutika sind für die Selbstmedikation freigegeben und damit nicht erstattungsfähig.

- Prof. Dr. med. Karin Kraft, Rostock 